\title{
Paradoxical Regulation of Human Argininosuccinate Synthetase cDNA Minigene in Opposition to Endogenous Gene: Evidence for Intragenic Control Sequences
}

\author{
Frederick M. Boyce III, Robert J. Pogulis, and Svend O. Freytag \\ Department of Biological Chemistry and Program in Cellular and Molecular Biology, University of Michigan Med- \\ ical School, Ann Arbor, Michigan 48109-0606
}

Received 21 June 1988-Final 28 October 1988

\begin{abstract}
Human somatic cell variants resistant to the arginine analog, canavanine, express 200-fold increased levels of argininosuccinate synthetase (AS) $m R N A$ as compared to parental cells. In this study we examined whether $A S$ cDNA sequences contain cis-acting regulatory elements that are involved in the induction of $A S M R N A$ in canavanine-resistant cells. Minigene constructs containing $A S c D N A$ sequences under the transcriptional control of a viral promoter were stably transfected into the human squamous cell carcinoma line, RPMI 2650. Upon conversion of cells to canavanine-resistance, expression of the endogenous AS gene increased by two orders of magnitude as expected. Surprisingly, however, expression of AS cDNA minigenes decreased 10- to 15-fold in canavanine-resistant cell variants. The observed down-modulation of $A S$ cDNA minigene expression was dependent upon a concomitant induction of the endogenous AS gene and not simply expression of the canavanine-resistant phenotype. This paradoxical regulation was specific for AS gene sequences since a minigene containing the neomycin-resistance gene in place of $A S c D N A$ sequences failed to regulate. Furthermore, minigenes lacking a substantial portion of the AS cDNA also failed to exhibit the down-modulation. These findings suggest that expression of the human AS gene is regulated by a specific and limiting, positively-acting, trans-acting mechanism in canavanineresistant cells and that exogenous $A S$ cDNA (mRNA) sequences can compete for this mechanism.
\end{abstract}

\section{INTRODUCTION}

Rare variants of nonhepatic cells that express high levels of AS mRNA, equivalent to those in the liver, can be isolated by sclection in the presence of the arginine analog, canavanine (1). The human squamous cell carcinoma line, RPMI 2650, gives rise to variants at a frequency of $10^{-6}-10^{-7}$ when using micromolar concentrations of canavanine. Interestingly, these canavanine-resistant cell variants stably express 200 -fold increased levels of AS mRNA as compared to parental RPMI 2650 cells without amplification of $A S$ genomic sequences (1). Previous studies using $A S$ promoter-gene fusions showed that the human $A S$ promoter was not more transcriptionally active in canavanine-resistant cell variants when added exogenously $(2,3)$. These results raised the possibility that the regulatory elements that are necessary for the induction of AS mRNA in canavanine-resistant cells might be intragenic. This possibility is the subject of the present report. 


\section{MATERIALS AND METHODS}

Construction of RSV-AS Expression Plasmids. RSV-AS contains sequences from the AS cDNA, pAS419 (4), from the AvaI site at base 1 to the PvuII site at base 1510 (5). These sequences were cloned between the HindIII and BglII sites of RSV $\beta$-globin (6), after removal of the $\beta$-globin sequences. RSVAS $\Delta$ HindIII was constructed by digesting RSV-AS with HindIII, filling in the recessed ends with the Klenow subunit of DNA polymerase, followed by ligation with T4 DNA ligase. RSV-AS $\Delta$ HindIII was verified as being unable to produce a functional $A S$ gene product by transfection of Chinese hamster RJK88 cells followed by selection in medium lacking arginine but containing citrulline $($ DMEM $-\mathrm{a}+\mathrm{c})(2)$. RJK88 cells lack endogenous AS activity and will die in DMEM $-\mathrm{a}+\mathrm{c}$ unless complemented with an exogenous and functional $A S$ gene. RSV-AS $\Delta$ HindIII failed to yield colonies in this assay whereas the parental plasmid, RSV-AS, produced hundreds of colonies. RSV-AS $\Delta$ HindIII-ter was constructed as follows. The plasmid pAS412/pUC-8, which contains the 1.3-kb PstI cDNA insert of pAS412 (5) cloned into the PstI site of pUC-8, was digested with BamHI and HindIII. A 250-bp Bam HI-HindIII fragment, which contains the first 232 bp of the AS cDNA, was isolated, and the ends were made blunt with the Klenow subunit of DNA polymerase. This blunted fragment was then cloned between the HindIII and BgIII sites of RSV $\beta$-globin after removal of the $\beta$-globin sequences and blunting of the HindIII and BglII ends with the Klenow subunit of DNA polymerase.

Cell Culture and Plasmid DNA Transfections. The maintenance of cell lines in culture, selection of canavanine-resistant cell variants and plasmid DNA transfections were performed as previously described $(2,7)$. In a typical experiment, 10-20 canavanine-resistant colonies were obtained in three to four weeks from $1-2 \times 10^{7}$ cells. The cells were then pooled and grown en masse for preparation of RNA. Quantification of the endogenous AS RNA and RSV-AS RNA was performed within two to four weeks following pooling of canavanine-resistant colonies to minimize the outgrowth of nonrepresentative clones.

S1 Nuclease Assays. Total cellular RNA was isolated using the guanidinium isothiocyanate $\mathrm{CsCl}$ density gradient method (8). DNA probes for S1 nuclease assays were prepared by 5 '-end labeling the DNA using $\left[{ }^{32} \mathrm{P}\right] \gamma$-ATP and polynucleotide kinase (8). S1 nuclease assays were performed using total cellular RNA as previously described $(2,7)$. Reaction products were applied to $8 \%$ polyacrylamide sequencing gels and protected fragments were visualized by autoradiography.

\section{RESULTS}

To test the possibility that AS exon sequences were involved in the 200 -fold induction of AS mRNA in canavanine-resistant cells, we constructed the expression plasmid RSV-AS (Fig. 1). RSV-AS contains a nearly full-length AS CDNA positioned downstream of the Rous sarcoma virus (RSV) promoter. This cDNA lacks the first 27 bases of exon 1 and the last 37 bases of the terminal exon, which includes polyadenylation signal sequences. Splicing and polyadenylation functions are provided by the vector. Cloned RPMI 2650 cell lines stably expressing RSVAS were obtained by cotransfection of RSVAS plasmid DNA with the dominant selectable marker gene, $R S V$ neo (6). Cell lines were screened for expression of RSV-AS mRNA by $\mathrm{S} 1$ nuclease assays (data not shown), and canavanine-resistant variants for each expressing cell line were obtained by selection in media containing $3 \mu \mathrm{M}$ canavanine (2). Expression of the endogenous $A S$ gene and RSV-AS cDNA minigene in parental cells and in pooled canavanine-resistant cell lines was examined using quantitative S1 nuclease 


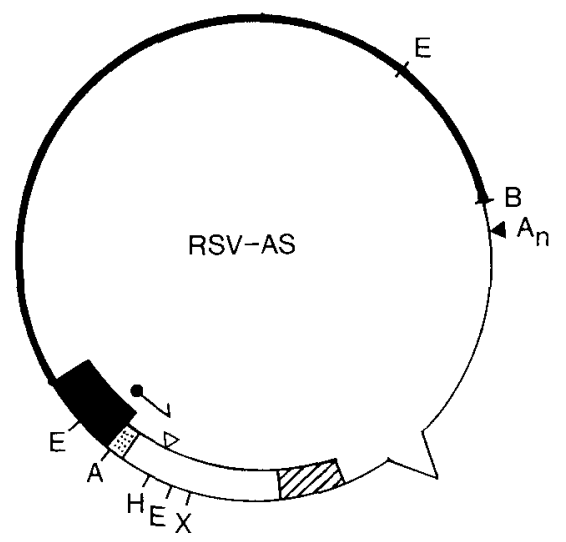

Fig. 1. Diagram of RSV-AS. Solid box, RSV promoter sequences; stippled box, AS 5'-untranslated sequences; open box, AS coding sequences; hatched box, AS 3'untranslated sequences; thin line, SV40 splicing and polyadenylation sequences; thick line, procaryotic vector sequences. The arrow indicates the transcription initiation site. A, AvaI; B, BamHI; H, HindIII; E, EcoRI; X, XhoI; $A_{n}$, polyadenylation site.

assays (Fig. 2). The endogenous AS RNA is alternatively spliced including or deleting exon 2 (4) and thus yields two protected fragments with the probe used in these assays. In cell line R30B4, which expressed relatively low levels of RSV-AS mRNA, expression of the endogenous $A S$ gene increased by two orders of magnitude upon conversion of cells to canavanine-resistance, as expected (Fig. 2, lanes $c$ and d). Surprisingly, however, we observed a concomitant 10- to 15-fold decrease in RSV-AS expression upon induction of the endogenous $A S$ gene in canavanine-resistance cells. The observed down-modulation was observed in two other independent cell lines that also expressed low levels of RSV-AS (data not shown). These findings indicated that expression of an AS CDNA minigene was regulated in a reciprocal fashion to the endogenous $A S$ gene and raised the possibility that the two $A S$ genes (mRNAs) were competing for a common regulatory mechanism in canavanine-resistant cells. In contrast, in cell line R30B2, which expressed high levels of RSV-AS mRNA, the endogenous AS mRNA was only minimally induced

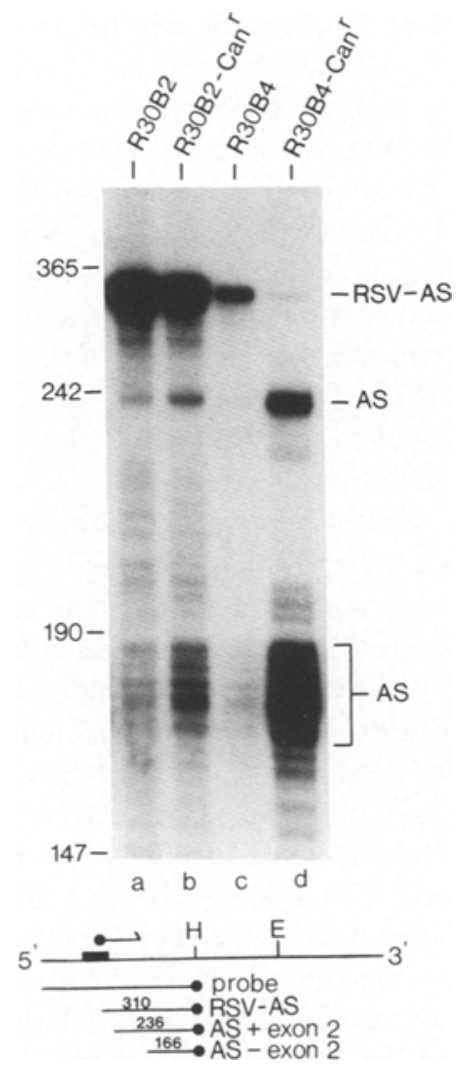

Fig. 2. Quantification of endogenous AS and AS cDNA minigene RNA using S1 nuclease analysis. Fifty micrograms of total cellular RNA were used in all assays. The probe was linearized RSV-AS $5^{\prime}$ end-labeled at the HindIII site in AS cDNA sequences. The endogenous AS mRNA lacking exon 2 gives a wide region of protection when using this probe and is probably the result of "nibbling" by S1 nuclease. The identities of the protected fragments are indicated. H, HindIII; E, EcoRI. The numbers on the left indicate the migration of DNA markers (MspI-digested pUC-9).

upon conversion of cells to canavanine-resistance, while RSV-AS expression was essentially unchanged (Fig. 2, lanes a and b). It is likely that the endogenonous AS mRNA was induced only slightly in cell line R30B 2 since these cells were partially resistant to canavanine, probably due to the high levels of RSVAS mRNA.

Other cell lines $(N>10)$ that acquired canavanine-resistance without inducing the endogenous $A S$ gene did not exhibit downmodulation of RSV-AS expression (unpub- 
lished data). Since these cell lines contain a full-length AS cDNA minigene that does not produce a functional AS enzyme (see below), they have acquired resistance to canavanine through an alternative mechanism that does not involve high $A S$ expression. Thus, it is clear that down-modulation of RSV-AS expression is not due to a culture artifact and requires concomitant induction of the endogenous $A S$ gene.

In order to be certain that the observed down-modulation of RSV-AS was not the result of selection pressures against recombinant AS enzyme activity, an additional cell line, R31A1, was constructed using an RSVAS minigene containing a frameshift mutation near the amino terminal end of the AS coding region (at amino acid position 53 , see open arrowhead Fig. 1). Pooled canavanineresistant variants of this cell line also showed a 10- to 15-fold decrease in RSV-AS expression concomitant with induction of the endogenous $A S$ gene (Fig. 3, lanes a and c). To rule out the possibility that this paradoxical response was due to a nonspecific down-modulation of cellular mRNAs in canavanine-resistant cells, we examined the expression of another cellular gene coding for triose phosphate isomerase (TPI) (9). The results show that expression of the TPI gene was unaffected upon conversion of cells to canavanine-resistance (Fig. 3, lanes a and $c$ ).

It was possible that the observed downmodulation of RSV-AS expression was due to a nonspecific effect on the viral promoter or polyadenylation sequences contained in these minigenes. Therefore, we examined the expression of another transfected gene, $R S V$ neo, which also utilizes the same RSV promoter and SV40 splicing and polyadenylation sequences contained in RSV-AS. The results show that unlike RSV-AS, expression of RSVneo was unaltered in canavanine-resistant variants of cell line R31A1 (Fig. 4A). To confirm that AS cDNA sequences were necessary for the observed down-modulation, a derivative of RSV-AS, RSV-AS $\Delta$ HindIII-

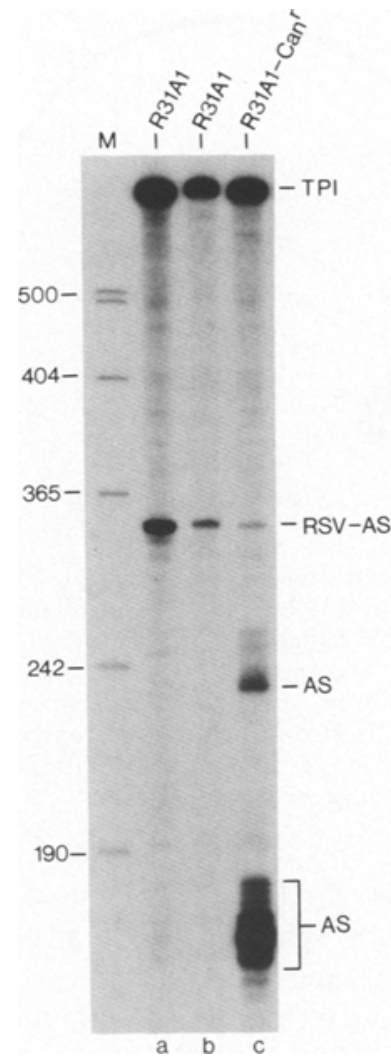

Fig. 3. S1 nuclease analysis showing down-modulation of RSV-AS $\Delta$ HindIII, a minigene containing a frameshift mutation. Lanes a and c, $50 \mu \mathrm{g}$ of total cellular RNA; lane $\mathrm{b}, 10 \mu \mathrm{g}$ of total cellular RNA. The probe used to detect the AS RNAs is the same as that described in the legend of Fig. 2. The probe used to detect triose phosphate isomerase (TPI) mRNA was a 936-bp EcoRI fragment of the human cDNA (9) $5^{\prime}$ end-labeled at the EcoRI sites.

ter, which lacks a substantial portion of the AS cDNA was constructed and tested. This minigene contains only the $5^{\prime}$ terminal 232 bases of the AS CDNA and lacks approximately 1300 bases of cDNA $3^{\prime}$ to the HindIII site (see Fig. 1). As before, this derivative was stably introduced into RPMI 2650 cells followed by selection of canavanine-resistant cell variants. Quantification of RNA in parental cells and canavanine-resistant cell variants revealed that RSV-AS $\Delta$ HindIII-ter mRNA was expressed equally in both cell types (Fig. 4B). Thus, the paradoxical down-modulation of RSV-AS expression in canavanine-resis- 


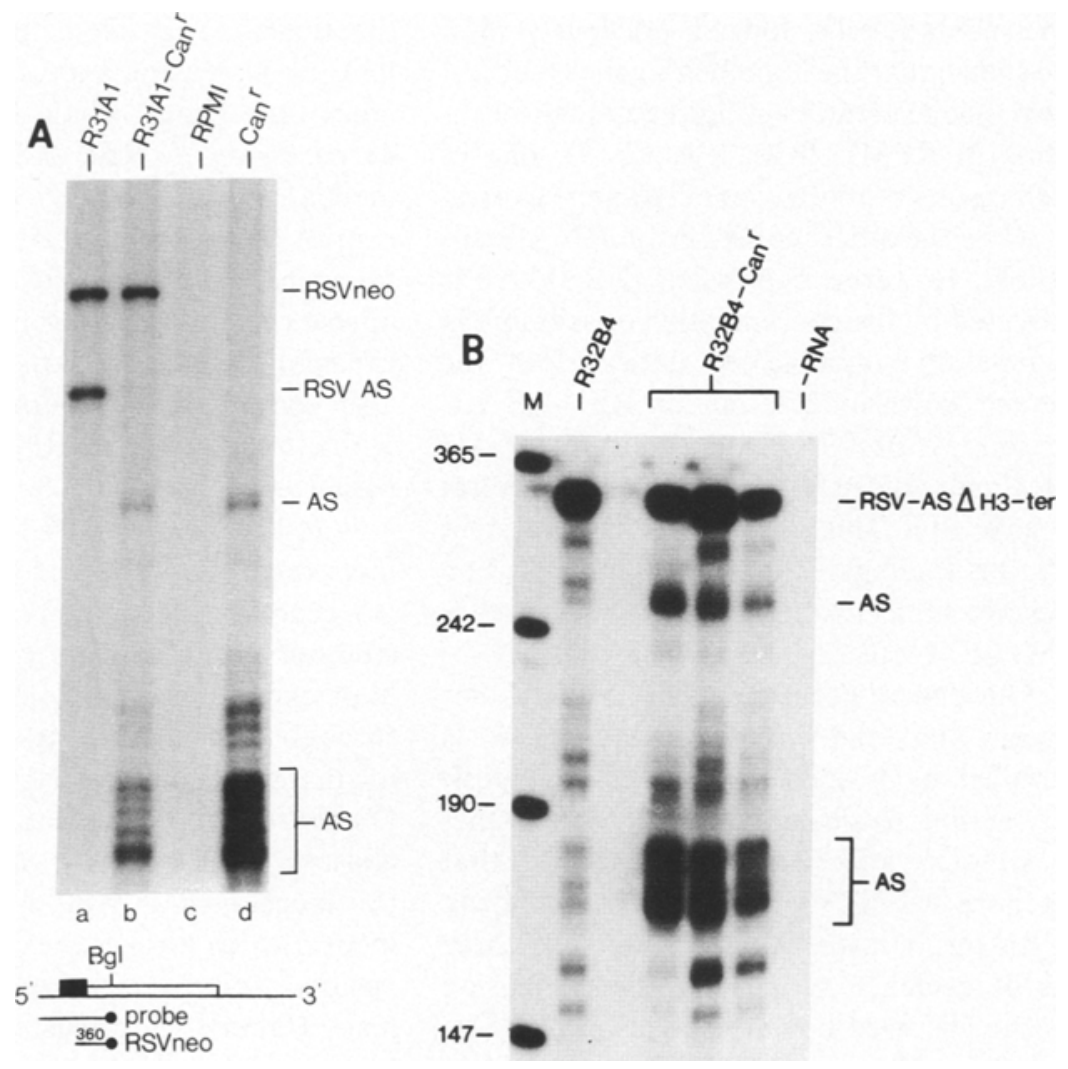

Fig. 4. (A) $\mathrm{S} 1$ nuclease analysis showing that the down-modulation is specific for AS sequences. Lanes a and $\mathrm{b}, 12 \mu \mathrm{g}$ of total cellular RNA; lanes c and d, $25 \mu \mathrm{g}$ of total cellular RNA. The probe used to detect the AS RNAs is the same as that described in the legend of Fig. 2. The probe used to detect RSVneo mRNA was RSVneo plasmid DNA (6) linearized with BglII and 5' end-labeled at the BglII sites. RPMI, RPMI 2650 cell RNA; Can ${ }^{\mathrm{r}}, \mathrm{Can}^{\mathrm{r}}-1$ cell RNA. (B) S1 nuclease analysis showing absence of down-modulation with a truncated derivative of RSV-AS. RSV-AS $\Delta$ HindIII-ter contains only those AS sequences $5^{\prime}$ to the HindIII site in the AS cDNA (see Fig. 1). Twenty-five micrograms of total cellular RNA were used in all assays. The probe used to detect the AS RNAs is the same as in Fig. 2. The results from three canavanine-resistant cell lines of $\mathrm{R} 32 \mathrm{~B} 4$ are shown.

tant cell variants is specific for AS cDNA sequences and the target sequences for this regulatory mechanism must lie $3^{\prime}$ to the HindIII site in the AS cDNA.

\section{DISCUSSION}

There are several possible mechanisms that would explain why RSV-AS expression is specifically regulated in opposition to the endogenous $A S$ gene upon conversion of cells to canavanine-resistance. For instance, one way to explain the observed reciprocal regulation is to hypothesize the existence of two separate regulatory mechanisms. The cDNA minigenes used in these studies are significantly different than the endogenous $A S$ gene in that they lack AS introns. Thus, one mechanism (factor) could interact with AS exon sequences and suppress AS mRNA expression, and a second more powerful mechanism could interact with $\mathrm{AS}$ intron sequences and increase AS mRNA expression. The net result on the endogenous $A S$ gene would be increased expression in the canavanine-resistant cell. In contrast, RSV-AS would only interact with the negative-acting mechanism and thus show decreased expression in canav- 
anine-resistant cells. Indeed, previous studies have shown that the human $A S$ gene is subject to two independently acting regulatory mechanisms in RPMI 2650 cells $(2,3)$, one of which results in repression of $A S$ gene expression while the other induces AS mRNA levels 200 -fold. However, expression of RSV-AS is unaffected by the concentration of arginine in the medium (unpublished data); thus, the observed down-modulation of RSV-AS expression is not the metabolite-mediated repression previously described for the natural $A S$ gene (1). This observation is consistent with the findings that $A S$ promoter-fusion genes are subject to repression by arginine in RPMI 2650 cells $(2,3)$.

Our data are consistent with a model that proposes that the endogenous $A S$ gene is controlled by a specific and limiting, positively-acting, trans-acting mechanism (factor) in canavanine-resistant cells and that exogenous AS cDNA sequences can compete for this mechanism. We have provided three lines of evidence supporting the conclusion that the observed down-modulation of RSV$\mathrm{AS}$ is specific for $A S$ gene sequences: (1) expression of another transfected gene, $R S V n e o$, which differs from RSV-AS only by substitution of neo sequences for AS sequences, does not exhibit the down-modulation; (2) expression of another cellular gene, $T P I$, is not subject to this regulation; and (3) deleting a portion of the AS cDNA from RSV-AS eliminates the down-modulation. The observation that the endogenous $A S$ gene and transfected cDNA minigenes are regulated in a reciprocal fashion suggests that the two AS genes (mRNAs) are competing for a common mechanism (factor). Thus, it follows that this mechanism (factor) must be limiting, otherwise the apparent competition would not have been observed. If this competition model is correct, the mechanism (factor) must be positive-acting since RSV-AS expression decreases concomitantly with increased expression of the endogenous $A S$ gene. This conclusion is based on the expectation that titration of a negative-acting mechanism (factor), by increasing expression of the endogenous $A S$ gene, would have resulted in derepression of RSV-AS expression. The mechanism is clearly trans-acting since expression of exogenously added AS cDNA minigenes is affected. Finally, the mechanism appears to be operating in the nucleus since canavanine-resistant variants of R30B2 cells also contain reduced amounts of RSV-AS RNA in the poly(A) - RNA fraction (unpublished observations).

Is this model consistent with all known facts regarding regulation of the endogenous $A S$ gene in canavanine-resistant cells? Other studies from this laboratory have shown that high expression of AS mRNA is mediated through a positive-acting mechanism operating in the nucleus of canavanine-resistant cells (7). Furthermore, studies using canavanineresistant lymphoblasts have indicated that this mechanism is trans-acting (2). Thus, our model, which invokes the existence of a single, positively-acting, trans-acting nuclear mechanism (factor), is consistent with all known facts regarding high $A S$ expression in canavanine-resistant cells. Our model requires that this mechanism (factor) be limiting, suggesting that its efficiency, but not its concentration, increases in canavanine-resistant cells. Therefore, the model invokes a change in the affinity of this mechanism (factor) for $A S$ gene (mRNA) sequences and that the affinity for endogenous $A S$ gene (mRNA) sequences is greater than the affinity for $R S V-A S$ gene (mRNA) sequences in canavanine-resistant cells. This mechanism (factor) could have different affinities for the endogenous $A S$ gene (mRNA) and $R S V-A S$ gene (mRNA) if it interacts with AS intron sequences that are absent from RSV-AS. Alternatively, it could recognize a unique secondary structure in the endogenous AS RNA. However, since minigenes containing only AS cDNA sequences are subject to the down-modulation, this mechanism (factor) must interact with AS exon sequences. It is important to note that 
this model makes no assumption whether the down-modulation involves $A S$ gene or RNA sequences and thus is consistent with a mechanism that acts either at the transcriptional or posttranscriptional level.

Support for this model will require mapping the sequences involved in this regulation and construction of RSV-AS derivatives containing AS intron sequences. Our task is made more difficult by the large size of the human $A S$ gene $(63 \mathrm{~kb})$ and the large number of introns (4). Nevertheless, it is clear that the human $A S$ gene is subject to a novel regulatory mechanism that warrants further investigation.

\section{ACKNOWLEDGMENTS}

RSV-AS was constructed by Tom Flickinger. F.M.B. was supported by a Burton Baker Memorial Fellowship from the Michigan Cancer Research Center and training grants in Cellular and Molecular Biology and the Medical Scientist Training Program. This research was supported by a grant awarded to S.O.F. from the American Cancer Society.

\section{LITERATURE CITED}

1. Su, T., Bock, H.-G., O'Brien, W., and Beaudet, A. (1981). J. Biol. Chem. 256:11826-11831.

2. Boyce, F., Anderson, G., Rusk, C., and Freytag, S. (1986). Mol. Cell. Biol. 6:1244-1252.

3. Jackson, M., O'Brien, W., and Beaudet, A. (1986). Mol. Cell. Biol. 6:2257-2261.

4. Freytag, S., Beaudet, A., Bock, H.-G., and O'Brien, W. (1984). Mol. Cell. Biol. 4:1978-1984.

5. Bock, H.-G., Su, T., O'Brien, W., and Beaudet, A. (1983). Nucleic Acids Res. 11:6505-6512.

6. Gorman, C., Padmanabhan, R., and Howard, B. (1983). Science 221:551-553.

7. Boyce, F., and Freytag, S. (1989). Somat. Cell Mol. Genet. 15:113-121.

8. Maniatis, T., Fritsch, E., and Sambrook, J. (1982). Molecular Cloning: A Laboratory Manual, (Cold Spring Harbor Press, Cold Spring Harbor, New York).

9. Maquat, L., Chilcote, R., and Ryan, P. (1985). J. Biol. Chem. 260:3748-3753. 